PSU-ASTR0 94/2-1

(February 1994)

\title{
Smoothed Particle Interpolation
}

\author{
Pablo Laguna \\ Department of Astronomy and Astrophysics \\ and \\ Center for Gravitational Physics and Geometry \\ The Pennsylvania State University \\ University Park, PA 16802
}

\begin{abstract}
Smoothed particle hydrodynamics (SPH) discretization techniques are generalized to develop a method, smoothed particle interpolation (SPI), for solving initial value problems of systems of non-hydrodynamical nature. Under this approach, SPH is viewed as strictly an interpolation scheme and, as such, suitable for solving general hyperbolic and parabolic equations. The SPI method is tested on (1) the wave equation with inhomogeneous sound speed and (2) Burgers equation. The efficiency of SPI is studied by comparing SPI solutions to those obtained with standard finite difference methods. It is shown that the power of SPI arises when the smoothing particles are free to move.
\end{abstract}

Subject headings: hydrodynamics - methods: numerical

*e-mail: pablo@astro.psu.edu 


\section{INTRODUCTION}

Perhaps one of the most powerful aspects in computational studies is the development of a fully adaptive scheme for solving partial differential equations. Without adaptive numerical schemes, current large memory and fast supercomputers are not capable yet of obtaining highly accurate solutions to physically interesting three-dimensional problems. For this reason, an optimal management of computational resources has been one of the driving forces in the effort to achieve both temporal and spatial adaptability as a way of obtaining a higher degree of accuracy.

The goal for reaching optimal temporal adaptability consists of designing algorithms that evolve each region of the computational domain with time-steps according to their local time-scales. In particle methods, local time-step algorithms are aimed at subsets of particles. The main difficulty here arises in designing a time evolution algorithm compatible with the neighbor-finding scheme (tree-code). In the case of finite differencing, the target of adaptability are subgrids. Each subgrid, for instance, is evolved according to its own Courant condition. Special attention must be paid at the subgrid boundaries where gridpoints in coarser meshes are not updated as often as in the finer meshes. Other approaches to temporal adaptability exploit multiple time-stepping applied at the equation instead of the domain level. These methods rely on the possibility of identifying in the evolution equations the slowly varying terms that require time-step $\Delta t$ in contrast to rapidly varying terms with time-step $\delta t<\Delta t$. The slowly varying terms are then computed once every large time-step $\Delta t$, and the rapidly varying terms are subcycled $\Delta t / \delta t$ times for each large time-step $\Delta t$. An example of this approach is called acoustic-subcycling, which has successfully improved the efficiency of compressible flow codes (O'Rourke \& Amsden 1986) when applied to low Mach number problems.

Technically speaking, spatial adaptability exhibits a higher degree of difficulty than temporal one. For finite difference methods, this translates into designing an efficient remeshing algorithm. Spatially adaptive finite difference methods for hyperbolic equations have been proposed by Berger and Oliger (1984) using multiple component grids. This approach was the keystone for Choptuik's (1993) finding of scaling and critical behavior in the gravitational collapse of a scalar field. Adaptive finite element methods, which have been mainly used for elliptic problems, have been also applied to hyperbolic equations (Davis \& Flaherty 1982). Finally, particle methods have been explicitly designed to exploit adaptability; a set of moving points carry the information of the dynamics of the system. Smoothed particle hydrodynamics (SPH) (for a review, see Benz 1990) is an example of such methods and constitutes the focus of this paper.

SPH has become a remarkably robust hydrodynamic method, in particular for astrophysical systems where vast range of scales are present. It has been successfully applied to stellar encounters(Davies, Benz, \& Hills 1991), i galaxy formation (Hernquist \& Katz 1989), tidal disruptions (Evans \& Kochanek 1989; Laguna, Miller, Zurek, \& Davies 1993), and more recently to problems outside the astrophysical domain, such as hypervelocity impacts. SPH has also been reformulated to describe relativistic fluids in curved space-times (Laguna, Miller \& Zurek 1993). SPH has been also used in combination with other methods in studying mixed systems where additional physics is required (hydrodynamics + magnetism, hydrodynamics + gravity, hydrodynamics + collisionless matter, etc.). In most of these cases, 
SPH is only applied to the hydrodynamic equations, and other numerical methods are used in the remaining set of equations. Mann (1993) has solved the relativistic, self-gravitating spherical collapse using finite element methods to compute the gravitational potentials and $\mathrm{SPH}$ for the hydrodynamics equations. In the study of large scale structure formation, SPH is combined with $N$-body methods to simulate hybrid systems of Hot $(\mathrm{SPH})+$ Cold $(N$ body) Dark Matter. There are, however, examples of studies in which, still in the presence of fluids, SPH has been applied to non-hydrodynamical equations. For instance, in the low frequency and infinite conductivity limit Stellingwerf and Peterkin (1989) have developed a simple and elegant method for smoothed particled magnetohydrodynamics.

The main goal of this paper is to address the following question: Can the success of SPH be extended to purely non-hydrodynamical problems? In other words, can one take advantage of SPH gridless nature of computing spatial derivatives and move particles with arbitrary "grid" velocity? We present one-dimensional results that point in the direction of an affirmative answer to this question. In Section 2 of the paper, we present the method for applying SPH discretization techniques to general hyperbolic and parabolic equations, giving special attention to truncation errors. Section 3 contains one-dimensional tests consisting of solutions to the wave equation with inhomogeneous sound speed; for these tests the particles are "fixed" in time (Eulerian framework). In Section 4, we repeat the tests of Section 3, but now we allow for particle motion (Lagrangian framework). Solutions to Burgers equation using SPH-type methods are obtained in Section 5. Finally, discussion and conclusions are given in Section 5.

\section{SPI: SMOOTHED PARTICLE INTERPOLATION}

The key ingredients of SPH are: (1) the fluid elements of the system are represented by particles, (2) the dynamics of the particles is governed by the hydrodynamic equations written in the Lagrangian form, and (3) the spatial derivatives at the particle positions are calculated via an interpolation kernel. Given a physical function $f(\mathbf{x})$ (pressure, density, etc.), its mean smoothed value can be obtained from

$$
\langle f(\mathbf{x})\rangle \equiv \int W\left(\mathbf{x}, \mathbf{x}^{\prime} ; h\right) f\left(\mathbf{x}^{\prime}\right) d^{3} x^{\prime}
$$

where $W\left(\mathbf{x}, \mathbf{x}^{\prime} ; h\right)$ is the kernel and $h$ the smoothing length. The kernel $W\left(\mathbf{x}, \mathbf{x}^{\prime} ; h\right)$ has compact support in a region of $O(h)$, and for hydrodynamic calculations the kernel is assumed to be of class $C^{1}$, i.e., its first derivatives exist and are continuous. One can show that this smoothing procedure is second-order accurate; that is, $\langle f(\mathbf{x})\rangle=f(\mathbf{x})+O\left(h^{2}\right)$. In particular, for the case of an spherically symmetric kernel the truncation errors due to the smoothing are

$$
\langle f(\mathbf{x})\rangle=f(\mathbf{x})+\alpha h^{2} \Delta f+O\left(h^{3}\right),
$$

where

$$
\alpha=\int W\left(\left|\mathbf{x}-\mathbf{x}^{\prime}\right| ; h\right)\left|\mathbf{x}-\mathbf{x}^{\prime}\right|^{2} h^{3} d^{3} x^{\prime}
$$


is independent of $h$; in the one-dimensional case, $\alpha=1 / 4$. The consistency requirement that $\langle f(\mathbf{x})\rangle \rightarrow f(\mathbf{x})$ as $h \rightarrow 0$, imposes the following normalization condition on $W$ :

$$
\int W\left(\mathbf{x}, \mathbf{x}^{\prime} ; h\right) d^{3} x=1
$$

The smoothing procedure (2.1) provides a natural prescription for calculating spatial derivatives at the particle locations. One has that, when ignoring surface terms, integration by parts of

$$
\langle\nabla f(\mathbf{x})\rangle=\int W\left(\mathbf{x}, \mathbf{x}^{\prime} ; h\right) \nabla^{\prime} f\left(\mathbf{x}^{\prime}\right) d^{3} x^{\prime}
$$

yields,

$$
\langle\nabla f(\mathbf{x})\rangle=\int f\left(\mathbf{x}^{\prime}\right) \nabla W\left(\mathbf{x}, \mathbf{x}^{\prime} ; h\right) d^{3} x^{\prime},
$$

where $\nabla^{\prime}$ and $\nabla$ are gradients with respect to $\mathbf{x}^{\prime}$ and $\mathbf{x}$, respectively. Equation (2.6) is just the statement that $\langle\nabla f(\mathbf{x})\rangle=\nabla\langle f(\mathbf{x})\rangle$. Similarly, it is not difficult to show that for a vector field $\mathbf{v}(\mathbf{x})$, the smoothed value of its divergence is given by $\langle\nabla \cdot \mathbf{v}(\mathbf{x})\rangle=\nabla \cdot\langle\mathbf{v}(\mathbf{x})\rangle$, or equivalently by

$$
\langle\nabla \cdot \mathbf{v}(\mathbf{x})\rangle=\int \mathbf{v}\left(\mathbf{x}^{\prime}\right) \cdot \nabla W\left(\mathbf{x}, \mathbf{x}^{\prime} ; h\right) d^{3} x^{\prime}
$$

Finally, the smoothing of $\nabla^{2} f$ is obtained by successively applying properties $\langle\nabla \cdot \mathbf{v}(\mathbf{x})\rangle=$ $\nabla \cdot\langle\mathbf{v}(\mathbf{x})\rangle$ and $\langle\nabla f(\mathbf{x})\rangle=\nabla\langle f(\mathbf{x})\rangle$. One gets that $\left\langle\nabla^{2} f\right\rangle=\nabla^{2}\langle f\rangle$, which has as integral representation

$$
\left\langle\nabla^{2} f\left(\mathbf{x}_{a}\right)\right\rangle=\int f\left(\mathbf{x}^{\prime}\right) \nabla^{2} W\left(\mathbf{x}, \mathbf{x}^{\prime} ; h\right) d^{3} x^{\prime} .
$$

One can show that the second-order accurate nature of the smoothing procedure is preserved under differentiation. The differentiation rules (2.6) and (2.7) to compute $\langle\nabla f(\mathbf{x})\rangle$ and $\langle\nabla \cdot \mathbf{v}(\mathbf{x})\rangle$, together with definition (2.1), is all that is needed to derive the SPH equations in their integral form (Benz 1990).

The next step is to obtain the discrete version of Eqs. (2.1) and (2.6)-(2.8); that is, a discrete representation of the integrals in those equations. In order to accomplish this, one defines first a number density distribution

$$
n(\mathbf{x}) \equiv \sum_{a=1}^{N} \delta\left(\mathbf{x}-\mathbf{x}_{a}\right)
$$

with $\left\{\mathbf{x}_{a}\right\}_{a=1, . ., N}$ the collection of $N$-points (particles) where the physical functions are known. The integrals in Eqs. (2.1) and (2.6)-(2.8) are evaluated by multiplying the integrands by $n(\mathbf{x}) /\langle n(\mathbf{x})\rangle=1+O\left(h^{2}\right)$, yielding

$$
\llbracket f\left(\mathbf{x}_{a}\right) \rrbracket=\sum_{b=1}^{N} \frac{f\left(\mathbf{x}_{b}\right)}{\left\langle n\left(\mathbf{x}_{b}\right)\right\rangle} W\left(\mathbf{x}_{a}, \mathbf{x}_{b} ; h\right),
$$




$$
\begin{aligned}
& \llbracket \nabla f\left(\mathbf{x}_{a}\right) \rrbracket=\sum_{b=1}^{N} \frac{f\left(\mathbf{x}_{b}\right)}{\left\langle n\left(\mathbf{x}_{b}\right)\right\rangle} \nabla_{\mathbf{x}_{a}} W\left(\mathbf{x}_{a}, \mathbf{x}_{b} ; h\right), \\
& \llbracket \nabla \cdot \mathbf{v}\left(\mathbf{x}_{a}\right) \rrbracket=\sum_{b=1}^{N} \frac{\mathbf{v}\left(\mathbf{x}_{b}\right)}{\left\langle n\left(\mathbf{x}_{b}\right)\right\rangle} \cdot \nabla_{\mathbf{x}_{a}} W\left(\mathbf{x}_{a}, \mathbf{x}_{b} ; h\right),
\end{aligned}
$$

and

$$
\llbracket \nabla^{2} f\left(\mathbf{x}_{a}\right) \rrbracket=\sum_{b=1}^{N} \frac{f\left(\mathbf{x}_{b}\right)}{\left\langle n\left(\mathbf{x}_{b}\right)\right\rangle} \nabla_{\mathbf{x}_{a}}^{2} W\left(\mathbf{x}_{a}, \mathbf{x}_{b} ; h\right) .
$$

respectively. The SPH equations, in their sum representation, can be directly derived by substitution of (2.10)-(2.12) into the hydrodynamic conservation laws written in a Lagrangian form (Benz 1990).

Higher order derivatives can be obtained following a procedure similar to that used to derive Eqs. (2.10)-(2.11). In principle, the only requirement needed is that a kernel of class $C^{n}$ is used in the smoothing of a $n$-order derivative. For instance, the smoothed approximation to the Laplacian operator, given by Eq. (2.13) requires a $C^{2}$ kernel, so the popular spline kernel (Monaghan \& Lattanzio 1985) used in SPH fails because of its discontinuous second derivatives. For this reason, we will use a gaussian kernel

$$
W(r, h)=\frac{1}{\pi^{d / 2} h^{d}} \begin{cases}\exp \left(-u^{2}\right) & \left(u<u_{o}\right) \\ 0 & \left(u>u_{o}\right)\end{cases}
$$

where $u \equiv\left|\mathbf{x}_{a}-\mathbf{x}_{b}\right| / h, d=1,2,3$ denotes the dimension of the problem, and $u_{o}$ the kernel's compact support radius (Figure 1). As we will see below, a disadvantage of this kernel is that it needs to be defined over a larger compact support, typically $u_{o} \sim 5$. In contrast, the spline's compact support radius is by definition $u_{o}=2$; hence, in general, using a gaussian kernel requires a larger number of neighbors to achieve similar accuracy as with the spline kernel.

It is important to notice that we have distinguished the smoothing procedure \langle\rangle from the discrete smoothing procedure 【』 to emphasize that there are in general two sources of truncation errors in approximating a function for its smoothed-discrete value; one error, the smoothing-error $(\langle\epsilon\rangle)$, arises from the smoothing procedure itself (see Eq. (2.2)), and a second error, the sum-error $(\llbracket \epsilon \rrbracket)$, is generated from the discretization of the integrals; that is

$$
\sum_{b=1}^{N} \frac{f\left(x_{b}\right)}{n\left(x_{b}\right)} \frac{d^{n}}{d x^{n}} W\left(x, x_{b} ; h\right)=\frac{d^{n}}{d x^{n}} f(x)+\langle\epsilon\rangle+\llbracket \epsilon \rrbracket .
$$

It is straightforward to show that for the one-dimensional gaussian kernel, the generalization of the smoothing-error (2.2) to $n$-order derivatives is

$$
\langle\epsilon\rangle=\frac{h^{2}}{4} \frac{d^{n+2}}{d x^{n+2}} f(x)+O\left(h^{3}\right) .
$$

The distribution of the particles plays a fundamental role in computing the truncation sum-error. If the particles have a chaotic distribution, the approximations (2.10)-(2.13) 
would be Monte Carlo estimates of the integrals in Eqs. (2.1) and (2.6)-(2.8), respectively. However, in SPH the particles do not follow a chaotic position distribution; they are not completely independent. On the other hand, they are not, in general, distributed in a regular configuration either. Nonetheless, for estimating sum-errors, we are going to assume a regular distribution of particles because it is in this case for which the minimum truncation sum-error is obtained. This will guarantee that features arising from the sum-error in regular particle distributions will also show up in more realistic configurations. For simplicity, we will only consider here the one-dimensional case.

Following a procedure similar to that of deriving truncation errors in standard quadrature formulas, it is not difficult to show that the leading truncation sum-error in (2.15) is given by

$$
\llbracket \epsilon \rrbracket=h^{-n}\left(\frac{\Delta x}{h}\right)^{2} f(x) \frac{d^{n+2}}{d u^{n+2}} w(u)+O\left((\Delta x / h)^{4} h^{-n}\right),
$$

where $\Delta x$ is the particle interseparation and $w(u) \equiv h W(u, h)$ only depends on $u$. Furthermore, $h / \Delta x=N_{1 / 2} / u_{o}$, where $u_{o}$ is the compact support radius and $N_{1 / 2}$ denotes the number of neighbors of a particle at each of its "sides" (Figure 1). It is important to notice from (2.17) that in the case of "pure" smoothing $(n=0)$, the sum-error only depends on $h / \Delta x \propto N_{1 / 2}$, the number of neighbors.

Convergence tests in SPH have been usually done by doubling the total number of particles; for our one-dimensional case, this implies $\Delta x^{(k)}=2^{-k} \Delta x_{o}$ with $k=0,1, \ldots$ and $\Delta x_{o}$ the spacing of the coarsest mesh. Little mention has been given, though, to how the number of neighbors is handled, i.e., the $h / \Delta x \propto N_{1 / 2} \rightarrow \infty$ limit. One has to remember that there are two competing errors in going from the continuum expressions to the discrete, smoothed approximations. The smoothing-error has a discretization scale given by the smoothing length $h$, see Eq. (2.16), and the sum-error depends, on the other hand, not only on the number of neighbors $h / \Delta x \propto N_{1 / 2}$ within the compact support of the kernel, but also on the smoothing length $h$ for terms involving differentiation, see Eq. (2.17) with $n>0$. Convergence tests have to satisfy the condition $h^{-n}(\Delta x / h)^{2} \rightarrow 0$ as $\Delta x$ and $h \rightarrow 0$. To illustrate this, we have computed the smoothed values of an analytic function and its smoothed first and second derivatives for different resolutions; that is, $\Delta x^{(k)}=2^{-k} \Delta x_{o}$ and $h^{(l)}=2^{-l} h_{o}$ with $k, l=1, . ., 4$. Figure 2 shows the results using $f(x)=\sin (x)$. The lines join points of constant $\Delta x$. As expected, the top two plots show the second order convergence rate of $\llbracket f(x) \rrbracket$. A similar second-order behavior is obtained for $\llbracket f^{\prime}(x) \rrbracket$ and $\llbracket f^{\prime \prime}(x) \rrbracket$ if $N_{1 / 2}>8$. However, for $N_{1 / 2}<8$, there seems to be an ill defined limit for $\llbracket f^{\prime}(x) \rrbracket$ and $\llbracket f^{\prime \prime}(x) \rrbracket$ when $h \rightarrow 0$; there the errors grow as $h \rightarrow 0$. As mentioned before, the answer to this puzzle is in how the limit $h^{-n}(\Delta x / h)^{2}$ is taken. We see that in particular the limit used in Fig. 2, $\Delta x, h \rightarrow 0$ with $h / \Delta x \propto N_{1 / 2}$ constant, will break down for $N_{1 / 2}<8$. Of course in practice, it is impossible to take the limit to the continuum; however, it is important to have, at a given resolution scale, enough number of neighbors, so the smoothing procedure falls within the second-order regime of the truncation errors. In our one-dimensional case, this means to have $N_{1 / 2}>8$.

Finally, in SPH the particles are identified with fluid elements; hence, their dynamics is governed by the hydrodynamic equations. In generalizing SPH methods beyond the scope of hydrodynamics, we view SPH as strictly an interpolation scheme (Smoothed Particle Interpolation: SPI) and, as such, used only to compute spatial gradients without the need of 
introducing a grid. The particles could be either "fixed" in space, although not necessarily uniformly distributed, or free to move. The latter case will represent a truly adaptive implementation. For the moving particles (Lagrangian) case, a rule that dictates motion of the particles should be provided. In principle, the rule for moving particles can be arbitrarily chosen; however, it is not clear at this stage whether non-single valued velocity fields are recommended since they could possibly lead to difficulties such as the one arising in hydrodynamic calculations when particle penetration occurs (Monaghan 1989).

\section{EULERIAN SPI}

In this section we will concentrate our attention on testing only the interpolating features of SPI. Hence, the interpolating particles will remain fixed in their original positions through out the evolution; the adaptive (moving particles) properties of SPI will be addressed in the next section. For these fixed particle tests, we start by considering the wave equation

$$
\partial_{t}^{2} \phi(\mathbf{x})-c^{2}(\mathbf{x}) \nabla^{2} \phi(\mathbf{x})=0,
$$

where $\partial_{t}^{2} \equiv \frac{\partial^{2}}{\partial t^{2}}$. We define the "momentum" variable $\chi \equiv \partial_{t} \phi$, and rewrite Eq. (3.1) as

$$
\partial_{t} \phi=\chi
$$

and

$$
\partial_{t} \chi=c^{2} \nabla^{2} \phi
$$

We now apply smoothing to (3.2) and (3.3). It is important to keep in mind that the smoothing properties act only on spatial directions; hence, temporal differentiation and smoothing commute, $\left\langle\partial_{t} f\right\rangle=\partial_{t}\langle f\rangle$. For temporal discretization, we use standard finite difference schemes. From substituting (2.10) and (2.13) into Eqs. (3.2) and (3.3), one obtains the discrete SPI approximations to Eqs. (3.2) and (3.3) to be

$$
\frac{\phi_{a}^{n+1}-\phi_{a}^{n}}{\Delta t}=\chi_{a}^{n+1 / 2}
$$

and

$$
\frac{\chi_{a}^{n+3 / 2}-\chi_{a}^{n+1 / 2}}{\Delta t}=c_{a}^{2} \sum_{b=1}^{N} \frac{\phi_{b}^{n+1}}{n_{b}} \nabla_{a}^{2} W_{a b},
$$

where $W_{a b} \equiv W\left(\mathbf{x}_{a}, \mathbf{x}_{b} ; h\right)$. The time step is given by $\Delta t=\operatorname{Dh} / \max \left(c_{s}(x)\right)$ with $D<\sqrt{3} / 2$. It is evident from these expressions that temporal integration is performed using a leap frog method (Roache 1985). There are two interlaced time-steps (integer- and half-steps). The terms $\partial_{t} \chi, \phi$ and spatial derivatives of $\phi$ are placed at integer-steps; similarly, $\partial_{t} \phi, \chi$ and spatial derivatives $\chi$ are placed at half time-steps. This temporal discretization makes the numerical integration second-order accurate.

To investigate the effects of the numerical dispersion in SPI, we evolved a gaussian pulse through a homogeneous medium; $c(x)=1$ in Eq. (3.2). The initial conditions for $\phi$ and its "momentum" $\chi$ were

$$
\phi(x)=\phi_{o} \exp \left[-k^{2}(x-t)^{2}\right]
$$


and

$$
\chi(x)=2 k^{2}(x-t) \phi(x),
$$

respectively. Solution errors are presented as cumulative relative errors; that is,

$$
\epsilon=\left(\frac{\sum_{a=1}^{N}\left(\widetilde{\phi}_{a}-\phi_{a}\right)^{2}}{\sum_{a=1}^{N} \widetilde{\phi}_{a}^{2}}\right)^{1 / 2}
$$

where $\phi$ and $\widetilde{\phi}$ are the numerical and analytical solutions, respectively. Figure (3.a) shows the solution error as a function of the resolution scale following a displacement of four gaussian width-lengths. The second order convergence rate implied by Eq. (2.2) is evident from this figure. For comparison, in Fig. (3.b) we plot the difference between SPI and FD solution errors; the error differences are plotted as a function of resolution scale. The resolution scale is defined as the discretization scale (particle interseparation in SPI or mesh spacing in FD) for which both methods, SPI and FD, yield the same absolute truncation error. It is not difficult to show by substituting (2.14) into Eq. (2.2), that for uniformly spaced particles given a grid spacing $\Delta x(F D)$ in FD, a smoothing length of $h=\Delta x(F D) / \sqrt{3}$ yields the same SPI and FD truncation errors. Furthermore, one also gets that the ratio of SPI particle spacing to FD grid spacing is given by $\Delta x(F D) / \Delta x(S P I)=\sqrt{3} N_{1 / 2} / u_{o}$, where $N_{1 / 2}$ is the number of neighbors at either side of each particle and $u_{o}$ the radius of the support of the kernel in smoothing length units. As we mentioned before, typically $u_{o} \sim 5$ and $N_{1 / 2} \sim 8$; therefore, $\Delta x(F D) / \Delta x(S P I) \sim 2.8$. That is, for each FD grid point, we need almost three SPI particles to achieve the same absolute solution error. However, it is important to emphasize that both methods have the same convergence rate, namely $O\left(h^{2}\right)$ for SPI and $O\left(\Delta x^{2}\right)$ for FD.

The next test constists of a one-dimensional gaussian pulse propagating through a medium in which the sound speed has a jump at the origin. That is,

$$
c(x)=c_{o} \frac{[3+\tanh (x / \sigma)]}{2}
$$

where, for simplicity, we use $c_{o}=1$, and $\sigma$ is chosen such that the jump is smooth over a few particles. The pulse has a gaussian profile as before, see Eq. (3.6), and is initially in the region $x<0$ where the sound speed is unity. Figure (4) shows four snapshots of the evolution. As expected, the pulse scatters at the point where the speed of sound has a jump. Two pulses emerge, one propagating in the same direction as the original pulse and a second one in the opposite direction. The calculation was repeated using a FD method; the bottom plot shows the difference between the SPI and FD solution for the last snapshot.

\section{LAGRANGIAN SPI}

We allow now for particle motions. However, for simplicity, we restrict ourselves to motion of particles in a constant velocity field. We start by rewriting equations (3.2) and (3.3) in the frame of reference $(t, \mathbf{x})$ comoving with the particles. The coordinate transformations between the comoving and laboratory frame $\left(t^{\prime}, \mathbf{x}^{\prime}\right)$ are $t=t^{\prime}$ and $\mathbf{x}=\mathbf{x}^{\prime}-\mathbf{v} t^{\prime}$, with $\mathbf{v}$ the 
particle velocity. To avoid complicated notation, we have redefined the prime coordinate system as the laboratory frame. In these new coordinates, Eqs. (3.2) and (3.3) take the form

$$
\partial_{t} \chi-\mathbf{v} \cdot \nabla \chi=c^{2} \nabla^{2} \phi
$$

and

$$
\partial_{t} \phi-\mathbf{v} \cdot \nabla \phi=\chi
$$

respectively. One immediately sees that a description from a frame of reference comoving with the particles introduces transport-like terms in the equations; that is, terms of the form $\mathbf{v} \cdot \nabla$ but with the "wrong" transport velocity sign. These transport-like terms require the same careful consideration to prevent the development of instabilities as that given to transport terms in the density, energy and momentum equations in fluid dynamics. In addition to developing a stable form of the equations, a "correct" centering of each term in the equations must be implemented in order to preserve the second-order nature of the scheme.

Let us look first at Eq. (4.2). As mentioned in the previous section, in a leap-frog integration method, spatial derivatives of $\nabla \phi$ are placed at integer-steps; on the other hand, $\partial_{t} \phi$ and $\chi$ are located at half-steps. Hence, an approximation to $\nabla \phi$ at half time-steps is needed. We achieve this with the following second-order interpolation

$$
\phi^{n+1 / 2}=\frac{3 \phi^{n}-\phi^{n-1}}{2},
$$

with similar interpolation for $\chi$; that is, it is necessary to save previous time-step values of $\phi$ and $\chi$.

Using (2.10), (2.11) and (2.13), one obtains the smoothed approximations of Eqs. (4.1) and $(4.2)$ to be

$$
\frac{\phi_{a}^{n+1}-\phi_{a}^{n}}{\Delta t}=\sum_{b=1}^{N} \frac{\phi_{b}^{n+1 / 2}}{n_{b}} \mathbf{v}_{a} \cdot \nabla_{a} W_{a b}+\beta v_{a}^{2} \Delta t \sum_{b=1}^{N} \frac{\phi_{b}^{n+1 / 2}}{n_{b}} \nabla_{a}^{2} W_{a b}+\chi_{a}
$$

and

$$
\frac{\chi_{a}^{n+3 / 2}-\chi_{a}^{n+1 / 2}}{\Delta t}=\sum_{b=1}^{N} \frac{\chi_{b}^{n+1}}{n_{b}} \mathbf{v}_{a} \cdot \nabla_{a} W_{a b}+c_{a}^{2} \sum_{b=1}^{N} \frac{\phi_{b}^{n+1}}{n_{b}} \nabla_{a}^{2} W_{a b},
$$

respectively. We have added to (4.1) a diffusion term, second term in the r.h.s. of Eq. (4.4), of the form $\beta v^{2} \Delta t \nabla^{2} \phi$ with $\beta$ a dimensionless parameter of order unity. Without this numerical dissipation the equations are correctly centered but unstable.

We repeated the simulation in Sec. 3 of propagating a pulse in a medium with a homogeneous speed of sound, $c(x)=1$ everywhere, but now the particle velocity is $v=c$; the pulse remains stationary in the particle frame. Because of the artificial dissipation term in Eq. (4.4), the numerical solutions will drift from the analytic solutions of the continuum wave equation. Since we are interested for the moment in studying the convergence rate of SPI, we have added in Eq. (4.4) a source term $-\beta v^{2} \Delta t \nabla^{2} \widetilde{\phi}$ with $\widetilde{\phi}$ the analytic solution. With this term, Eqs. (4.4) and (4.5) have as analytic solution the gaussian pulse (3.6). This 
allows a direct estimate of the solution error (Choptuik 1986) from Eq. (3.8). We performed a series of runs with smoothing lengths $h^{(k)} \equiv 2^{-k} h_{o}, k=1, . ., 4$, where $h_{o}$ is the smoothing length of the coarsest level. The convergence factor of the solution error at the $k$ refinement level is defined as (Choptuik 1991)

$$
\xi^{(k)} \equiv \frac{\epsilon^{(k)}}{\epsilon^{(k+1)}},
$$

where $\epsilon^{(k)}$ is the solution error (See Eq. 3.8) at level $k$. Figure (5.a) shows the convergence factor $\xi^{(k)}$ of the solution error as a function of time. The solution error obtained at the end of the runs after the pulse has traveled 3 width-lengths $(t \approx 60)$ is plotted in Fig. (5.b). The solution errors suggest a convergence rate $O\left(h^{1.39}\right)$. This convergence rate implies that $\xi^{(k)} \rightarrow 2.62$ as $h \rightarrow 0$, which is consistent with the behavior of $\xi^{(k)}$ in Fig. (5.a).

Finally, we considered again the case in which the speed of sound has a jump given by (3.9); the speed of the particles was set to $v=1$, and the source term $-\beta v^{2} \Delta t \nabla^{2} \widetilde{\phi}$ of the previous test was not included. Figure (6) shows snapshots at the same time intervals as in Fig. (4); for comparison, in last plot of Fig. (6), solution differences between the moving and fixed particle cases are presented.

\section{SPI AND BURGERS EQUATION}

It is important to study the stability behavior of SPI discretization for nonlinear equations. For this purpose, we consider now Burgers equation, which reads

$$
\partial_{t} \phi+\phi \partial_{x} \phi=\alpha \partial_{x}^{2} \phi
$$

Due to the parabolic nature of Burgers equation, the temporal integration used in previous sections to handle the wave equation becomes unstable when applied to this case. For the sake of simplicity, we will consider first the advection-diffusion equation which is obtained from Burgers equation after replacing $\phi \partial_{x} \phi$ by $v \partial_{x} \phi$. Here again one needs to be careful with the correct centering of each of the terms in the equation. However, correct centering does not guarantee stability; it is well known that a centered-space and centered-time discretization of the advection-diffusion equation, although second-order accurate, is unstable. A simple cure, at the expense of sacrificing second-order accuracy, is obtained by using forward-time, centered-space scheme. We are interested, nonetheless, in obtaining a scheme as close as possible to second-order accuracy and achieving, at the same time, stability.

Our approach to solve the advective-diffusion equation is analogous to the FD scheme that combines leapfrog advection differences $\left(O\left(\Delta t^{2}, \Delta x^{2}\right)\right)$ with forward-time, centeredspace differencing of diffusion terms $\left(O\left(\Delta t, \Delta x^{2}\right)\right.$ ) (Roache 1985). For $\alpha \propto O(\Delta t)$, this method is $\left(O\left(\Delta t^{2}, \Delta x^{2}\right)\right)$. Under this method, the advection diffusion equation has a SPI approximation

$$
\frac{\phi_{a}^{n+1}-\phi_{a}^{n-1}}{\Delta t}=-v_{a} \sum_{b=1}^{N} \frac{\phi_{b}^{n}}{n_{b}} \partial_{x_{a}} W_{a b}+\alpha \sum_{b=1}^{N} \frac{\phi_{b}^{n-1}}{n_{b}} \partial_{x_{a}}^{2} W_{a b} .
$$

As with the previous section, to study the convergence rate, we have added a source term to $(5.2)$ of the form $-\alpha \partial_{x}^{2} \widetilde{\phi}$, so an analytic solution to this modified advection-diffusion 
equation is given by the gaussian pulse (3.6). Figure (7.a) shows the convergence factor $\xi^{(k)}$ of the solution error. In addition, the solution errors after the pulse has traversed three times its original thickness are plotted as a function of the particle separation in Fig. (7.b) for $v=1$ and $\alpha=1$. These solution errors imply a $O\left(h^{1.95}\right)$ scheme and $\xi^{(k)} \rightarrow 4$ as $h \rightarrow 0$.

A similar procedure to that used for the advection-diffusion equation was applied to Burgers equation. The stable SPI representation of Burgers equation is given by

$$
\frac{\phi_{a}^{n+1}-\phi_{a}^{n-1}}{\Delta t}=-\phi_{a}^{n} \sum_{b=1}^{N} \frac{\phi_{b}^{n}}{n_{b}} \partial_{x_{a}} W_{a b}+\alpha \sum_{b=1}^{N} \frac{\phi_{b}^{n-1}}{n_{b}} \partial_{x_{a}}^{2} W_{a b} .
$$

Here again, to test the order of the method, we added a term $\widetilde{\phi} \partial_{x} \widetilde{\phi}-\alpha \partial_{x}^{2} \widetilde{\phi}$, so the equation has an analytic solution $\widetilde{\phi}$ given by the pulse (3.6). The convergence factor $\xi^{(k)}$ of the solution error is plotted in Fig (8.a) and the final solution error in Fig. (8.b) for $\alpha=0.2$. We find that the SPI discretization (5.3) to Burgers equation gives solution error of $O\left(h^{1.98}\right)$. Finally, in Figure (9) we show the solution to Burgers equation, without the source terms $\widetilde{\phi} \partial_{x} \widetilde{\phi}-\alpha \partial_{x}^{2} \widetilde{\phi}$, for an initially gaussian pulse. As expected, the pulse develops shock-like features due to the non-linear term and also diffuses because of the dissipation term.

\section{CONCLUSIONS}

We have presented a method, smoothed particle interpolations (SPI), based on SPH techniques to solve hyperbolic and parabolic equations of non-hydrodynamic nature. The method was tested solving the one-dimensional wave equation with inhomogeneous sound speed and Burgers equation. We showed that the intrinsic adaptive nature of SPH is directly carried over. We also provided a prescription for handling advection-type terms that arise when particles are free to move or when dealing with Burgers equation. Furthermore, we showed the feasibility of obtaining $O\left(h^{2}\right)$ schemes under SPI discretizations.

A possible drawback of this approach is its particle requirements compared with the mesh-point cost in finite difference methods. We have shown and tested that, for uniformly spaced particles, SPI required a minimum of three smoothing particles for each grid point in FD in order to achieve equivalent absolute solution errors.

In principle, generalization of this work to two or three dimensional systems should be straightforward (Laguna 1994). After all, one of the reasons for SPH's popularity has been its simplicity for implementation in higher dimensions. Moreover, all the technology developed in N-body methods and standard $\mathrm{SPH}$ for finding neighboring particles can be directly utilized.

\section{ACKNOWLEDGMENTS}

I thank Richard Matzner and Matt Choptuik for numerous discussions and helpful suggestions. Work supported in part by the NASA (at Los Alamos National Laboratory), NSF Young Investigator award PHY-9357219, and NSF grant PHY-9309834. 


\section{REFERENCES}

Benz, W. 1990, in Numerical Modeling of Stellar Pulsation: Problems E Prospects, ed. J.R. Buchler, (Dordrecht: Kluwer Academic), p269

Berger, M.J., \& Oliger, J. 1984, J. Comput. Phys., 53, 484

Choptuik, M. (1986) Ph.D. thesis, University of British Columbia

Choptuik, M. 1991, Phys. Rev. D, 44, 3124

Choptuik, M. 1993, Phys. Rev. Lett., 70, 9

Davies, M.B., Benz, W. \& Hills, J. 1991, Astrophys. J., 381, 449

Davis, S., \& Flaherty, J. 1982, SIAM J. Sci. Statist. Comput., 3, 6,

Evans, C. R. \& Kochanek, C. S. 1989, Astrophys. J., 346, L13

Hawley, J. F., Smarr, L. L., \& Wilson, J. R. 1984, Astrophys. J., 277, 296

Hernquist, L., \& Katz, N. 1989, Astrophys. J., 70, 419

Laguna, P., Miller, W.A., \& Zurek, W. H. 1993, Astrophys. J., 404, 678

Laguna, P., Miller, W.A., Zurek, W.H., \& Davies, M.B. 1993, Astrophys. J., 410, L83

Laguna, P. 1993, in preparation

Mann, P.J. 1993, J. Comput. Phys., 107, 188.

Monaghan, J. J. 1989, J. Comput. Phys., 82, 1

Monaghan, J. J., \& Lattanzio, J. C. 1985, Astron. Astrophys., 149, 135

O’Rourke, P.J., \& Amsden, A.A. 1986, Los Alamos National Laboratory report LA-10849MS

Roache, P.J. 1985 Computational Fluid Dynamics, (Albuquerque: Hermosa Publishers)

Stellingwerf, R.F., \& Peterkin, R.E. 1989, Mission Research Corporation report MRC/ABQ$\mathrm{R}-1248$ 


\section{FIGURE CAPTIONS}

Fig.1 Gaussian kernel with compact support radius $u_{o}=5$. The compact support radius is defined as $u_{o}=N_{1 / 2} \Delta x / h$ where $h$ is the smoothing length, $\Delta x$ the particle interseparation and $N_{1 / 2}=10$ the number of neighbors of a particle at each of its sides. In the figure, the particle positions are denoted by open circles.

Fig.2 Smoothed values errors in the representation of $f(x)=\sin (x)$ and its smoothed first and second derivatives for $\Delta x^{(k)}=2^{-k} \Delta x_{o}, k=1$ (circle),2(cross), 3(triangle), 4(square) and $h^{(l)}=2^{-l} h_{o}, l=1,2,3,4$.

Fig.3 Results from a gaussian pulse propagating in a medium with uniform sound speed $c=1$. The particles were kept fixed throughout the evolution. (a) shows solution errors as a function of the particle interseparation. (b) shows the differences between SPI and FD solution errors. The errors were obtained after a pulse displacement of four initial gaussian width-lengths.

Fig.4 Propagation of a gaussian pulse through a medium in which the sound speed has a jump at the origin given by Eq. (3.9). The particles were kept fixed throughout the evolution. Bottom plot shows the difference between the SPI and FD solution in last snapshot.

Fig.5 Results from propagating a gaussian pulse in a medium with uniform sound speed $c=1$. The particles moved with speed $v=1$. (a) shows the convergence factor $\xi^{(k)}$ of the solution error. (b) shows the solution errors as a function of the particle interseparation at a pulse displacement of four pulse width-lengths.

Fig.6 Same as in Fig. (3) but for particles moving with speed $v=1$. The plot at the bottom in this case shows the differences of the solutions between the fixed and moving particle cases at the end of the run.

Fig.7 (a) Convergence factor $\xi^{(k)}$ of the solution error for the advective-diffusion equation. A source term of the form $-\alpha \partial_{x}^{2} \widetilde{\phi}$ was added to the equation in order to have a gaussian pulse as analytic solution. (b) Solution errors as a function of the particle interseparation.

Fig.8 Same as in Fig. (6) but for Burgers equation with a source term $\widetilde{\phi} \partial_{x} \widetilde{\phi}-\alpha \partial_{x}^{2} \widetilde{\phi}$.

Fig.9 Solution to Burgers equation of an initial gaussian pulse with $\alpha=0.2$. 
This figure "fig1-1.png" is available in "png" format from: http://arxiv.org/ps/astro-ph/9402062v1 
This figure "fig2-1.png" is available in "png" format from: http://arxiv.org/ps/astro-ph/9402062v1 
This figure "fig1-2.png" is available in "png" format from: http://arxiv.org/ps/astro-ph/9402062v1 
This figure "fig2-2.png" is available in "png" format from: http://arxiv.org/ps/astro-ph/9402062v1 
This figure "fig1-3.png" is available in "png" format from: http://arxiv.org/ps/astro-ph/9402062v1 